\title{
Endovascular management of two episodes of late intraperitoneal hemorrhage following laparoscopic gastrectomy for gastric cancer
}

\author{
QIANG HUANG, KUN GAO and REN-YOU ZHAI \\ Department of Interventional Radiology, Beijing Chaoyang Hospital, Capital Medical University, \\ Chaoyang, Beijing 100020, P.R. China
}

Received September 4, 2013; Accepted March 4, 2014

DOI: $10.3892 / \mathrm{mco} .2014 .288$

\begin{abstract}
Endovascular treatment for hemorrhagic complications following surgery has recently gained wide acceptance due to its minimal invasiveness compared to surgery. A 56-year-old male patient underwent laparoscopic gastrectomy for gastric cancer. There were two episodes of late intraperitoneal hemorrhage and endovascular treatment was performed. Transcatheter coil embolization of the gastroduodenal artery stump was successful in controlling the bleeding initially; however, hemorrhage recurred 7 days later. Repeated angiography revealed an obvious hemorrhage from the right gastric artery stump and embolization of the hepatic artery proper was performed to achieve immediate hemostasis. The endovascular treatment process was analyzed and the literature on similar situations was reviewed. In the present case, endovascular procedures were performed successfully to control bleeding in two episodes of late intraperitoneal hemorrhage. Angiography is recommended as the first-line modality for late intraperitoneal hemorrhage following laparoscopic gastrectomy and transcatheter coil embolization of the hepatic artery proper is safe and effective in selected cases.
\end{abstract}

\section{Introduction}

Late intraperitoneal hemorrhage is a potentially life-threatening complication following laparoscopic surgery and requires timely and precise identification of its source for definitive treatment. Endovascular treatment has recently gained wide acceptance due to its minimal invasiveness compared to surgery (1). In this study, we present a rare

Correspondence to: Dr Qiang Huang, Department of Interventional Radiology, Chaoyang Hospital, Capital Medical University, 8 Gongren Tiyuchang Nanlu, Chaoyang, Beijing 100020, P.R. China

E-mail: hq0713@163.com

Key words: intraperitoneal hemorrhage, laparoscopic gastrectomy, embolization case of two episodes of intraperitoneal hemorrhage from the gastroduodenal artery stump and the right gastric artery stump, respectively. Endovascular treatment was performed twice to achieve hemostasis. The patient recovered well after the second interventional procedure with coil embolization of the hepatic artery proper. The interventional procedure of angiography and coil embolization was reviewed and discussed.

\section{Case report}

A 56-year-old male patient underwent laparoscopic gastrectomy for gastric cancer. The postoperative period was uneventful until sudden onset of epigastric pain 45 days after the surgery. Following readmission, emergency computed tomography (CT) detected a hemoperitoneum (Fig. 1). The patient underwent urgent exploratory laparotomy. The abdominal exploration revealed moderate hemoperitoneum (300 $\mathrm{ml})$ but the culprit vessel was not identified. An indwelling catheter was placed into the abdominal cavity. The hematocrit of the patient continued to decrease according to the hemogram and hemorrhagic fluid was persistently drained from the catheter after laparotomy. Therefore, the patient was referred to our department for interventional treatment. Angiography and embolization was performed after obtaining written informed consent from the patient and his family.

Via the right femoral arterial approach, a 5-F catheter (Yashiro; Terumo, Tokyo, Japan) was placed through a $5-\mathrm{F}$ sheath and positioned in the celiac artery. The celiac arteriogram confirmed aneurysmal dilatation of the gastroduodenal artery stump (Fig. 2). There was no obvious active extravasation from the gastroduodenal artery stump; however, there was a distinct decrease in the hemoglobin concentration of $\geq 30 \mathrm{~g} / \mathrm{l}$. Therefore, we decided to perform the embolization. A coaxial technique was used to insert a 2.9-F microcatheter (Progreat ${ }^{\mathrm{TM}}$; Terumo) into the sac and microcoils (Tornado Embolization Microcoil ${ }^{\mathrm{TM}}$; Cook, Bloomington, IN, USA) were deployed to fill the sac and the gastroduodenal artery stump. A celiac artery angiogram after the embolization confirmed the successful occlusion of the lesion and the patency of the hepatic artery proper (Fig. 3). The right hepatic artery was visualized through selective superior mesenteric arteriography and superselective 

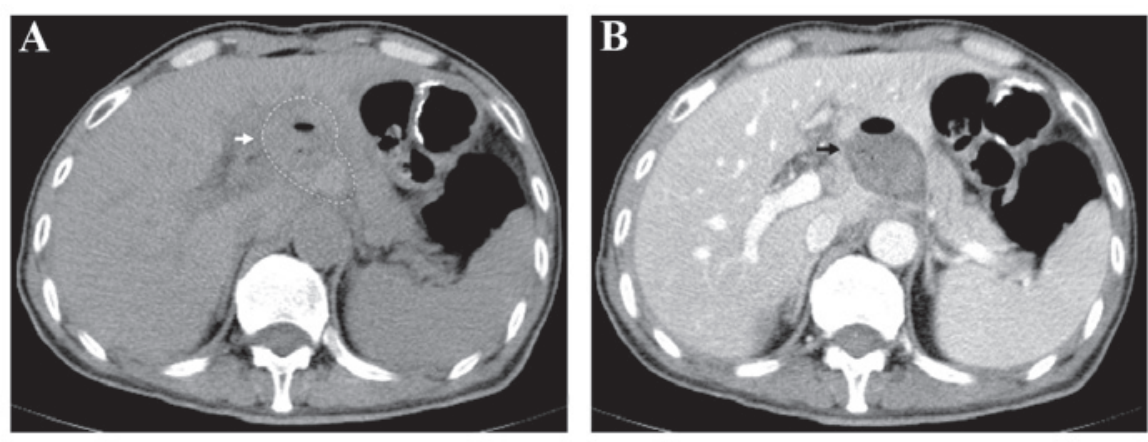

Figure 1. (A) Hemoperitoneum (white arrow) detected by routine computed tomography (CT) (B) Hemoperitoneum (black arrow) demonstrated distinctly by contrast-enhanced CT.

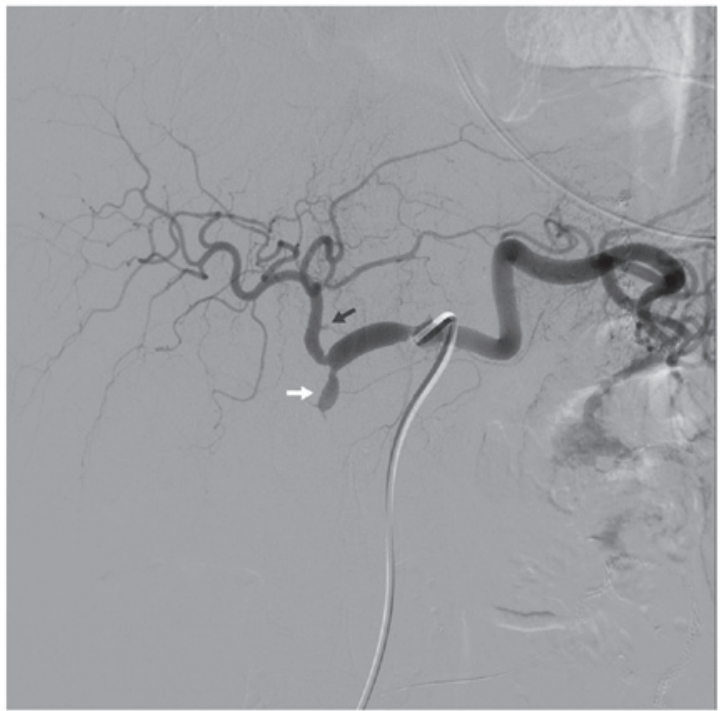

Figure 2. A celiac arteriogram confirmed the aneurysmal dilatation of the gastroduodenal artery stump (white arrow); the right gastric artery stump (black arrow) was identified, without contrast agent extravasation.

angiogram due to collateral flow via the pancreaticoduodenal arcade without filling of the sac (Fig. 4).

The bleeding ceased, with no hemorrhagic fluid in the indwelling catheter, and the patient recovered well after the embolization. However, a second episode of intraperitoneal hemorrhage occurred 7 days after the first interventional procedure. This time the patient was hemodynamically unstable, with $>400 \mathrm{ml}$ blood drained from the indwelling catheter. Urgent endovascular management was attempted again to locate and control the bleeding. The celiac arteriogram confirmed a well-embolized gastroduodenal artery stump and active extravasation from the right gastric artery stump (Fig. 5). However, the right gastric artery stump was too short to be embolized through catheterization. Following a discussion on the therapeutic options between the surgeons and interventional radiologists, relaparotomy was considered, although it would not be possible to control the bleeding without ligating the hepatic artery proper; furthermore, there was a minimal possibility of liver ischemia due to the collateral circulation from the superior mesenteric artery. Therefore, embolization of the hepatic artery proper distal as well as proximal to the right gastric artery stump was performed with

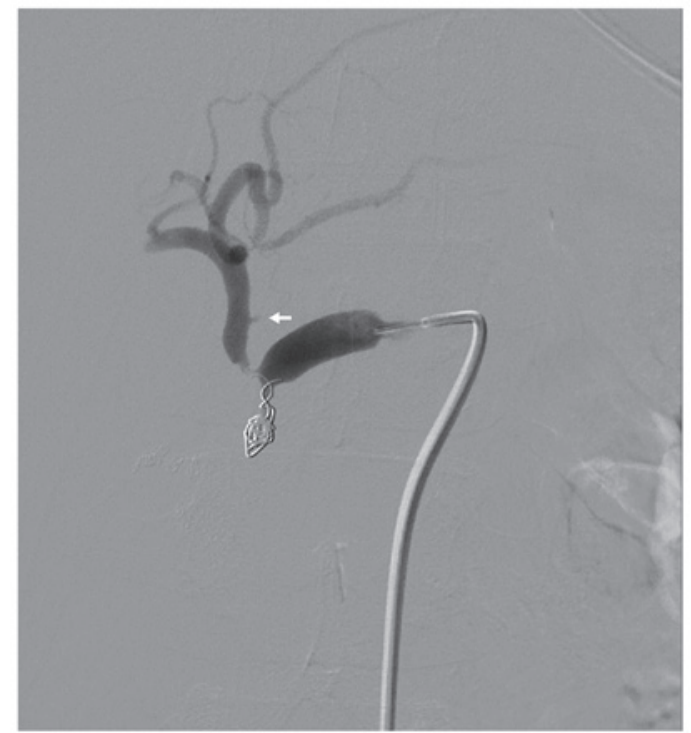

Figure 3. The celiac artery angiogram after embolization confirmed the successful occlusion of the gastroduodenal artery stump, with the normal right gastric artery (white arrow). There was vasospasm at the proximal part of the hepatic artery proper.

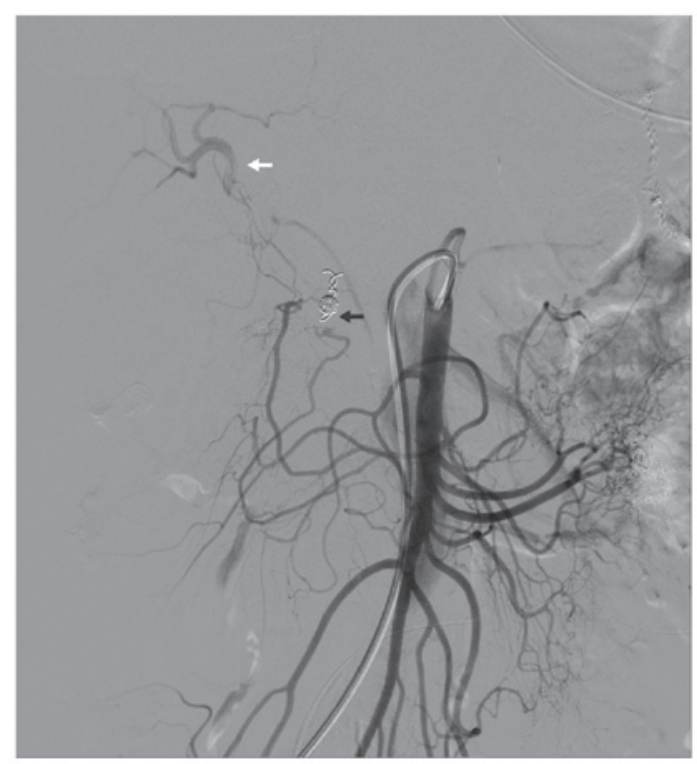

Figure 4. There was no filling of the embolized sac (black arrow) on the selective superior mesenteric arteriogram. The right hepatic artery (white arrow) was visualized through the collateral flow via the pancreaticoduodenal arcade. 


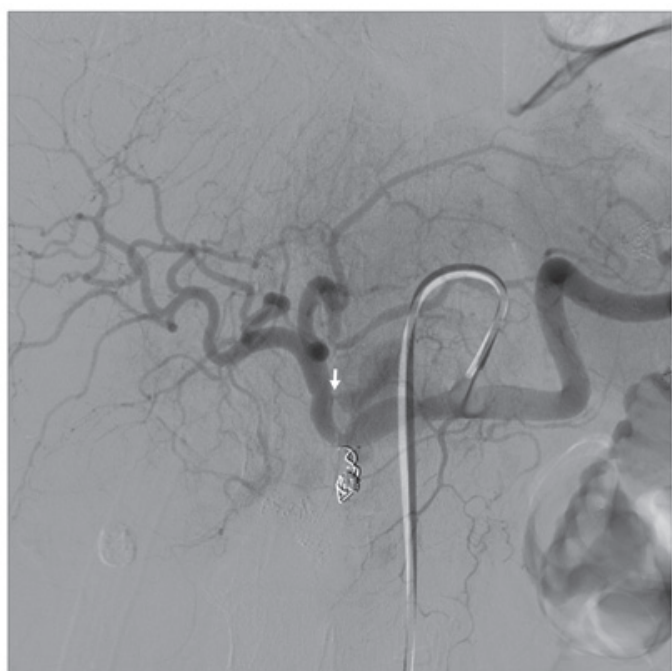

Figure 5. The celiac arteriogram confirmed a well-embolized gastroduodenal artery stump, while active extravasation was present from the right gastric artery stump (white arrow).

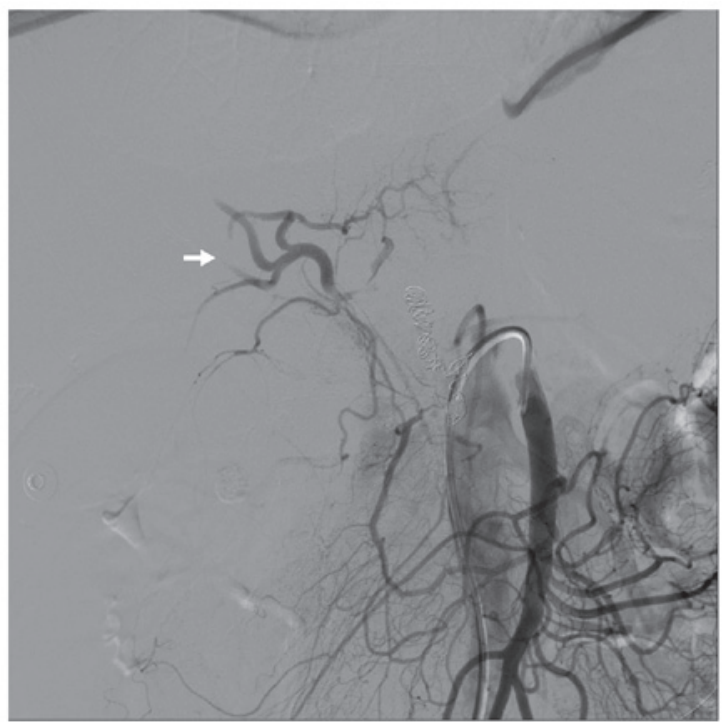

Figure 6. Filling of the right hepatic artery (white arrow) via the collateral pathway of the superior mesenteric artery was confirmed on the post-embolization angiogram.

microcoils. The completion celiac angiography confirmed immediate hemostasis and occlusion of the hepatic artery proper. Filling of the right hepatic artery via the collateral pathway of superior mesenteric artery was demonstrated by post-embolization angiography (Fig. 6).

The patient quickly regained hemodynamic stability after the second endovascular procedure. There was a transient mild elevation in the serum transaminases, but the enzyme levels returned to normal within 10 days, with no evidence of severe liver insufficiency or ischemia. A contrast-enhanced CT obtained 3 days after the second embolization confirmed good perfusion of the liver (Fig. 7). The hemoglobin level was stable (88-96 g/l) following the second embolization procedure and there was little hemorrhagic fluid drained from the intraperitoneal indwelling

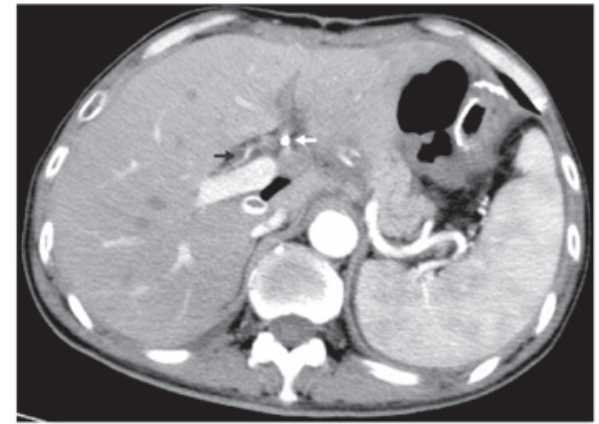

Figure 7. Contrast-enhanced CT obtained 3 days after the second embolization revealed good perfusion of the liver. The microcoils (white arrow) placed in the hepatic artery proper and the right hepatic artery (black arrow) are identified. CT, computed tomography.

catheter. The patient was discharged 20 days after the second interventional procedure.

\section{Discussion}

Laparoscopic gastrectomy is an alternative operative treatment modality that is widely accepted due to its minimal invasiveness compared to open gastrectomy (2-4). Postoperative bleeding is one of the most common operation-related severe complications mentioned in the literature $(2,5)$. Immediate and accurate diagnosis is essential in order to prevent a massive and life-threatening delayed hemorrhage. The traditional approach to late hemorrhage following abdominal surgery is reoperation; however, the mortality rates associated with reintervention are high (6). Furthermore, it is difficult to determine the precise source of the bleeding, due to the surrounding large amount of clotted blood. Definitive treatment may be more difficult in certain cases, even when the precise bleeding site has been located. Endovascular procedures provide a safe and effective method for diagnostic and therapeutic purposes and are widely used in similar situations, such as hemorrhage following pancreaticoduodenectomy (1). In fact, late intraperitoneal hemorrhage occurred more frequently following pancreaticoduodenectomy and endovascular procedures have been proven to be effective (1).

Delayed hemorrhage has been defined differently in the literature, ranging between 1 and 5 or more postoperative days $(6,7)$. The duration of 45 days between the laparoscopic gastrectomy and the onset of hemorrhage is relatively long. The titanium clamp was displaced postoperatively and was considered to be the cause of the delayed hemorrhage from the right gastric artery. Approximately $80 \%$ of the right gastric artery originates from the proximal branches of the hepatic trunk (8). The anatomical characteristics necessitate the occlusion of the hepatic trunk when selective embolization of the right gastric artery is not feasible. Moreover, the right gastric artery stump was too short for further ligation or coil embolization in this case, which made it impossible to maintain hepatic blood flow while controlling the bleeding by laparoscopy or open surgery.

CT angiography (CTA) is widely applied; however, we recommend angiography as the first-line modality for 
intraperitoneal hemorrhage following surgery. Selective visceral angiography is accurate in detecting the source of hemorrhage that would otherwise be undetectable with exploratory laparotomy. The greatest advantage of selective visceral angiography over CTA is that endovascular treatment may be performed in the same setting.

Liver failure following common hepatic artery embolization was feared, as fatal liver failure was previously reported following embolization of the common hepatic artery (7). However, similar situations were reported to be safe in the currently available literature $(1,9)$. The risk of liver failure is relatively low due to the dual blood supply from the portal and the arterial circulation (6). Collateral vessels are also important in maintaining liver perfusion, as in this case.

It was previously reported that stent grafts allow for excluding hemorrhagic lesions without compromising blood flow to the liver $(6,10)$. The use of a stent graft was proposed to avoid the potentially severe complications of arterial occlusion (6). However, anticoagulation should be administered to prevent thrombus formation following stenting, which was considered unacceptable by the surgeons for fear of recurrence of bleeding from other lesions in this case.

In conclusion, we recommend angiography as a first-line modality for late intraperitoneal hemorrhage following laparoscopic gastrectomy and transcatheter coil embolization of the hepatic artery proper is safe and effective in selected cases.

\section{References}

1. Yamashita Y, Taketomi A, Fukuzawa K, et al: Risk factors and management of delayed intraperitoneal hemorrhage after pancreatic and biliary surgery. Am J Surg 193: 454-459, 2007.

2. Kodera Y, Fujiwara M, Ohashi N, et al: Laparoscopic surgery for gastric cancer: a collective review with meta-analysis of randomized trial. J Am Coll Surg 211: 677-686, 2010.

3. Lee SW, Nomura E, Bouras G, et al: Long-term oncologic outcomes from laparoscopic gastrectomy for gastric cancer: a single-center experience of 601 consecutive resections. J Am Coll Surg 211: 33-40, 2010.

4. Ohtani H, Tamamori Y, Noguchi K, et al: Meta-analysis of laparoscopy-assisted and open distal gastrectomy for gastric cancer. J Surg Res 171: 479-485, 2011.

5. Bo T,Zhihong P, Peiwu Y, et al: General complications following laparoscopic-assisted gastrectomy and analysis of techniques to manage them. Surg Endosc 23: 1860-1865, 2009.

6. Boufi M, Hashemi AA, Azghari A, et al: Endovascular management of severe bleeding after major abdominal surgery. Ann Vasc Surg 27: 1098-1104, 2013.

7. Choi SH, Moon HJ, Heo JS, et al: Delayed hemorrhage after pancreaticoduodenectomy. J Am Coll Surg 199: 186-191, 2004.

8. Lee SW, Shinohara H, Matsuki M, et al: Preoperative simulation of vascular anatomy by three-dimensional computed tomography imaging in laparoscopic gastric cancer surgery. J Am Coll Surg 197: 927-936, 2003.

9. Hur S, Yoon CJ, Kang S-G, et al: Transcatheter arterial embolization of gastroduodenal artery stump pseudoaneurysms after pancreaticoduodenectomy: safety and efficacy of two embolization techniques. J Vasc Interv Radiol 22: 294-301, 2011.

10. Pasklinsky G, Gasparis A, Labropoulos N, et al: Endovascular covered stenting for visceral artery pseudoaneurysm rupture: report of 2 cases and a summary of the disease process and treatment options. Vasc Endovascular Surg 42: 601-606, 2008. 